\title{
ARTICLE
}

\section{Impact of adult attention deficit hyperactivity disorder and medication status on sleep/wake behavior and molecular circadian rhythms}

\author{
A. N. Coogan ${ }^{1}$, M. Schenk ${ }^{2}$, D. Palm², A. Uzoni (iD ${ }^{2}$, J. Grube $^{3}$, A. H. Tsang ${ }^{3}$, I. Kolbe ${ }^{3}$, N. M. McGowan ${ }^{1,4}$, R. Wandschneider ${ }^{2}$, M. Colla $^{2}$, \\ H. Oster ${ }^{3}$, J. Thome ${ }^{2}$ and F. Faltraco ${ }^{2}$
}

Attention deficit hyperactivity disorder (ADHD) is a common neuropsychiatric condition that has been strongly associated with changes in sleep and circadian rhythms. Circadian rhythms are near 24-h cycles that are primarily generated by an endogenous circadian timekeeping system, encoded at the molecular level by a panel of clock genes. Stimulant and non-stimulant medication used in the management of ADHD has been shown to potentially impact on circadian processes and their behavioral outputs. In the current study, we have analyzed circadian rhythms in daily activity and sleep, and the circadian gene expression in a cohort of healthy controls $(N=22)$, ADHD participants not using ADHD-medication $(N=17)$, and participants with ADHD and current use of ADHD medication $(N=17)$. Rhythms of sleep/wake behavior were assessed via wrist-worn actigraphy, whilst rhythms of circadian gene expression were assessed ex-vivo in primary human-derived dermal fibroblast cultures. Behavioral data indicate that patients with ADHD using ADHD-medication have lower relative amplitudes of diurnal activity rhythms, lower sleep efficiency, more nocturnal activity but not more nocturnal wakenings than both controls and ADHD participants without medication. At the molecular level, there were alterations in the expression of PER2 and CRY1 between ADHD individuals with no medication compared to medicated ADHD patients or controls, whilst CLOCK expression was altered in patients with ADHD and current medication. Analysis of fibroblasts transfected with a BMAL1:luc reporter showed changes in the timing of the peak expression across the three groups. Taken together, these data support the contention that both ADHD and medication status impact on circadian processes.

Neuropsychopharmacology (2019) 44:1198-1206; https://doi.org/10.1038/s41386-019-0327-6

\section{INTRODUCTION}

Attention deficit hyperactivity disorder (ADHD) is a common neuropsychiatric condition, with prevalence in adults currently estimated at $\sim 5 \%$ worldwide [1], and a persistence rate from the childhood condition in the order of $40-50 \%$ [2]. Aside from the core symptoms of increased impulsivity, attentional difficulties, and hyperactivity, sleep disturbances occur very frequently in adult ADHD [3]. The nature of the relationship between ADHD and sleep problems is not well understood, but in the general population sleep disturbance can lead to inattention [4] and impulsivity [5, 6], and as such disordered sleep may contribute to symptom severity in ADHD. Further, stimulant and non-stimulant medication used to manage ADHD in adults may themselves adversely impact sleep [7].

Sleep is a complex physiological process, with a number of definable stages that most likely serve a range of key functions within the brain and the rest of the body [8]. A vital regulator of sleep/wake behavior is the circadian clock [9]. The circadian system's interaction with the homeostatic drive to sleep in determining the timing of sleep and wake behavior is articulated in the two process model of sleep regulation [10]. The circadian system is an endogenous network that imposes a daily temporal architecture on physiology, and drives near 24-h rhythms in a host of molecular, endocrine, neural, and behavioral outcomes [11]. The molecular basis of the circadian clock is a panel of circadian clock genes, which form transcriptional -translational feedback loops regulating their own expression [12]. These clock genes also exert a widespread influence on gene expression across the vast majority of tissue and cell types, with $40 \%$ of all transcripts showing rhythmic expression in at least one tissue in the mouse [13]. Under normal circumstances, in order to maintain biological salience the internal clock is synchronized ("entrained") to appropriate environmental time cues, the most important such stimulus being light [14]. Altered entrainment of the circadian clock has been described in a number of psychiatric conditions, such as major depression and bipolar disorder [15]. Circadian rhythms have previously been shown to be altered in adult ADHD [16]. These alterations include dampening of rhythms of clock gene expression [17], increased eveningness in ADHD [18], and delayed dim-light melatonin onset [19].

Circadian changes associated with ADHD may be important inand-of-themselves through resultant changes in temporal

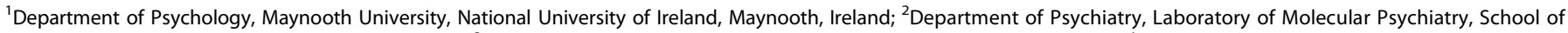

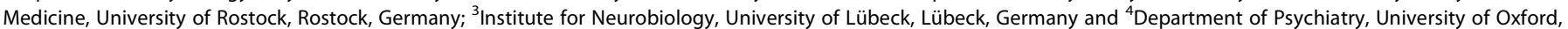
Oxford, UK

Correspondence: J Thome (johannes.thome@med.uni-rostock.de.de)

Received: 11 October 2018 Revised: 28 December 2018 Accepted: 22 January 2019

Published online: 6 February 2019 
Table 1. Clinical and demographic characteristics of the study sample

\begin{tabular}{|c|c|c|c|}
\hline & $\begin{array}{l}\text { Controls } \\
n=22\end{array}$ & $\begin{array}{l}\text { ADHD without } \\
\text { medication } \\
n=17\end{array}$ & $\begin{array}{l}\text { ADHD with } \\
\text { medication } \\
n=17\end{array}$ \\
\hline Age [years] & $38.1 \pm 12.2$ & $37.1 \pm 9.97$ & $39.3 \pm 10.9$ \\
\hline Female & $13(52 \%)$ & $7(41.2 \%)$ & $9(52.9 \%)$ \\
\hline Height $[\mathrm{cm}]$ & $175.1 \pm 8.2$ & $172.6 \pm 10.3$ & $172.5 \pm 7.1$ \\
\hline Weight [kg] & $77.4 \pm 17.3$ & $74.8 \pm 11.91$ & $73.9 \pm 13.4$ \\
\hline BMI & $25.2 \pm 4.37$ & $25.06 \pm 3.07$ & $24.95 \pm 4.83$ \\
\hline Employed/retired & $21 / 1$ & $17 / 0$ & $16 / 1$ \\
\hline IQ & $116 \pm 13.4$ & $107.8 \pm 10.9$ & $109.1 \pm 13.0$ \\
\hline WURSk score & $12.3 \pm 9.2$ & $35.8 \pm 10.6^{* * *, 5}$ & $46.4 \pm 11.2^{* * *}$ \\
\hline ADHD score & $5.3 \pm 3.8$ & $25.2 \pm 4.8^{* * *}$ & $26.12 \pm 7.48^{* * *}$ \\
\hline MEQ & $56.3 \pm 11.1$ & $43.3 \pm 11.1^{* * *}$ & $49.3 \pm 13.0$ \\
\hline $\begin{array}{l}\text { No psychiatric co- } \\
\text { morbidity }\end{array}$ & 25 (100\%) & $8(47 \%)$ & $9(53 \%)$ \\
\hline $\begin{array}{l}\text { Current } \\
\text { methylphenidate } \\
\text { usage }\end{array}$ & 0 & 0 & $14(83 \%)$ \\
\hline $\begin{array}{l}\text { Current atomoxetine } \\
\text { usage }\end{array}$ & 0 & 0 & $\begin{array}{l}4(23 \% ; N=1 \\
\text { for MPH + ATO } \\
\text { use) }\end{array}$ \\
\hline \multirow[t]{3}{*}{ ADHD subtype } & $\mathrm{n} / \mathrm{a}$ & 8 combined & 16 combined \\
\hline & & 3 hyperactive & 1 inattentive \\
\hline & & 6 inattentive & \\
\hline
\end{tabular}

Further clinical information is detailed in Supplementary Table 1 ${ }^{* *} P<0.01,{ }^{* * *} P<0.001$ and ${ }^{\$} P<0.05$

patterns of cognitive and executive function (as many such processes are under circadian control [20]). Further, circadian changes may impact through dysfunction of the sleep/wake cycle, with resulting daily impairments [8]. However, at present the directionality of the relationships between the circadian clock, sleep, and ADHD are unclear. For example, do altered circadian rhythms lead to disturbed sleep and ADHD symptoms, or does inattention/impulsivity/mind racing lead to sleep changes, which in turn lead to circadian changes? These questions are further complicated by the potential for ADHD medication, such as methylphenidate and atomoxetine, to alter circadian processes and sleep [21-24]. Indeed, sleep factors may moderate the clinical impact of ADHD medication [25]. To start to address these concerns, the current study examines circadian rhythms at the behavioral and molecular levels in patients with ADHD who are currently using ADHD medication, compared to healthy controls and to patients with ADHD who are not using ADHD medication.

\section{MATERIAL AND METHODS}

\section{Participant selection criteria}

Ethical approval for the conduct of the study, including obtaining skin samples and use of all human tissues, was given by the ethical review committee of Rostock University (Registration-number: A2013-159), and written informed consent was obtained from each study participant. The study was conducted according to the ethical guidelines of the Declaration of Helsinki.

Patients participating in the study were recruited via the Adult ADHD Outpatient Clinic of the Department of Psychiatry, Rostock University. All patients with ADHD were diagnosed by experienced psychiatrists in advance. Patients were made aware of the study through notices or by the study team directly contacting relevant patients. The healthy control group was made up of acquaintances of people involved in the study, advertised by word of mouth among employees of the clinic and employees of the Clinic for Psychiatry and Psychotherapy, Rostock. Only adult individuals, able to give informed consent, were included. Healthy controls without a history of childhood and adult ADHD or other significant diagnoses were matched for age and sex. Patients whose symptoms of possible comorbidities were more impairing than ADHD symptoms were excluded. Healthy controls were screened for ADHD using the WURS-k and the ASRS V1.1 screener to exclude subjects who showed evidence of ADHD in childhood or adulthood. Shift workers were also excluded. Three participants from the control group were ultimately excluded due to suspicion of a sleep disorder from inspection of actigraphy data. For the ADHD diagnosis, the WURS-k (Wender Utah Rating Scale) and the IDA (Integrated Diagnosis of Adult ADHD) were used. The IDA is composed of the ASRS V.1.1 (Adult ADHD Self Report Scale) and self-assessment of symptoms according to DSM-IV and ICD-10 criteria. The IQ of the healthy controls and ADHD groups was measured by using MWT-B (Multiple Selection Vocabulary Test). Detailed information about the psychometry is given in the Supplement. Details of the study sample can be found in Table 1 and Supplementary Table 1, 2 and 3.

Tissue isolation and fibroblast culture

Human dermal fibroblasts were isolated and cultured as described by Takashima [26].

For additional information on study design and cell culture, see Supplementary Material.

\section{Actigraphy}

In order to obtain objective measures of participants' sleep and circadian rhythm function, the rest-activity pattern of participants was recorded using wrist-worn actigraphs (Actiwatch 2, Philips Respironics, PA, USA). Actigraphs were worn on the non-dominant wrist for a period of at least 7 consecutive days. The recording interval of the device was set at $60-5$ epochs. Data occurring before the first and after the final midnight of each record were excluded, ensuring at least 6 complete days for each participant, with a complete weekend included in each record. Estimates of sleep/wake behavior were calculated using Actiware software version 5.57 (Philips Respironics, as above). The default medium activity threshold (40 activity counts) was selected for designating sleep from wake states as this threshold accords well with polysomnography and subjective sleep ratings [27].

Measurement of circadian gene expression

Upon confluency of the respective primary fibroblast cell culture from each participant, seven culture flask replicates were prepared and synchronized with $100 \mathrm{~nm}$ dexamethasone (Sigma-Aldrich, Darmstadt, Germany) for $30 \mathrm{~min}$. Samples were harvested every $4 \mathrm{~h}$ starting with time 0 after dexamethasone synchronization, rinsed with PBS (Gibco, Thermo Fisher, Paisley, UK), collected with solution $\mathrm{D}(4.5 \mathrm{M}$ guanidinium thiocyanate, $0.5 \%$ sodium- $\mathrm{N}$-lauryl sarcosine, $25 \mathrm{mM}$ tri-sodium citrate, $0.1 \mathrm{M}$ betamercaptoethanol) and frozen at $-70^{\circ} \mathrm{C}$. Later, total RNA was isolated by RNeasy Plus Mini Kit (Qiagen, Hilden, Germany) and subjected to reverse transcription with the use of Superscript III First-Strand Synthesis System (Invitrogen, Darmstadt, Germany). Gene expression of CLOCK, BMAL1, PER1, PER2, PER3, and CRY1 expression was measured by real-time quantitative reverse transcriptase-polymerase chain reaction (qRT-PCR) and analyzed for cycling behavior.

Viral infection, synchronization, and real-time bioluminescence monitoring

Primary fibroblast cell cultures from healthy control samples $(n=$ 7), ADHD patients currently using medication $(n=6)$ and ADHD patients not using ADHD medication $(n=6)$ were trypsinized (Gibco, Thermo Fisher, Paisley, UK), and $2.5 \times 10^{4}$ cells were seeded to a 96-well culture plate containing culture medium as 
described above. Viral transduction was conducted on the next day with $\sim 1.68 \times 10^{8}$ infection units (IFUs) of a plasmid expressing $B M A L$ 1:luc per dish in the presence of $8 \mu \mathrm{g} / \mathrm{mL}$ polybrene. The $B M A L 1:$ IUC lentiviral particles were produced from the HEK293T cells as previously described [28]. One day after transduction, cells were rinsed with PBS (Gibco, Thermo Fisher, Paisley, UK) and refreshed with culture medium. After 2 days, the cells were synchronized with $100 \mathrm{~nm}$ dexamethasone (Sigma Aldrich, Darmstadt, Germany) for $2 \mathrm{~h}$ and the medium was changed to DMEM without phenol red (Life Technologies, Darmstadt, Germany) containing 10\% fetal bovine serum, $25 \mathrm{mM}$ HEPES (Sigma Aldrich, Darmstadt, Germany), and $500 \mu \mathrm{M}$ beetle luciferin (Promega, Mannheim, Germany). Luminescence measurements were performed with a Berthold TriStar LB 941. The experiment was conducted for 7 days until rhythms dampened to flatness. Details of the study sample can be found in Table 1 and Supplementary Table 3.

\section{Statistical methods}

Circadian clock gene expression data were tested for significant circadian rhythmicity, using CircWave v. 1.4 software (generated by Dr. Roelof Hut; www.euclock.org) to determine the best-fitting linear harmonic regression with an assumed period of 24-h and with $a$ set at 0.05 . The center-of-gravity of each best-fitting waveform in CircWave was used as the circadian acrophase, and the associated estimation error was used as the SD. To obtain circadian parameters including phase, period length, rhythm amplitude, and damping rate, the LumiCycle Analysis program (Actimetrics) and Actogram J software was used. Luminometry data was detrended and smoothed with a rolling $3 \mathrm{~h}$ average as previously described $[29,30]$. Circadian parameters were derived from cosinor analysis and period was determined from Lomb-Scargle periodograms analysis.

Inferential statistics were carried out in either SPSS (IBM Corporation) or JASP Stats (https://jasp-stats.org/). Actigraphic data were analyzed via MANCOVAs, with age, sex and in some cases ADHD symptom severity included in the model as covariates. For MANCOVAs, Pillai's trace was used as the most robust statistic in the presence of inequalities of group sizes. qRT-PCR clock gene data were analyzed via mixed between-within ANOVAs, with age and sex included as co-variates. Phase data from both qRT-PCR and BMAL1:luc data were analyzed with circular statistics in the Oriana Program (Kovach Computing Services, UK). For all inferential tests, $P<0.05$ was deemed to indicate a statistically significant groupwise difference. For all ANOVAs, effects sizes are indicated with partial ETA squared, and interpreted according to Cohen [31]. Sample sizes were calculated via GPower software; for multivariate analysis the assumptions used were power of $0.8, a=0.05$ and 3 groups with 3 measures and it being important to detect medium effect sizes (Cohen's $f^{2}$ of 0.15 ), returning a required total sample size of 51 . For mixed between-within ANOVA analysis of clock gene data, using similar parameters for 3 groups and 7 within groups measurements indicated a required total sample of 48 .

\section{RESULTS}

Study sample

Table 1 and Supplementary Table 1, 2 and 3 describe the composition of the study sample. There were no significant differences in age, BMl, or sex across the three study groups, and the two ADHD groups did not vary in clinical severity of ADHD symptoms. MEQ scores indicated that un-medicated ADHD patients displayed more evening preference than controls, but ADHD patients with medication did not

\section{Actigraphy}

Measures from the non-parametric circadian rhythm analysis were analyzed across the three groups (controls, ADHD + medication, ADHD no medication) to examine circadian rhythm timing and robustness. Intradaily variability (IV), a measure of the within-day consolidation of rest-activity patterns, inter-daily stability (IS), a measure of variation in the activity rhythm across days, and relative amplitude (RA), a measure of rhythm fragmentation were assessed across the three groups in a MANCOVA, with age and sex serving as co-variates (Fig. 1a). There was a statistically significant
A

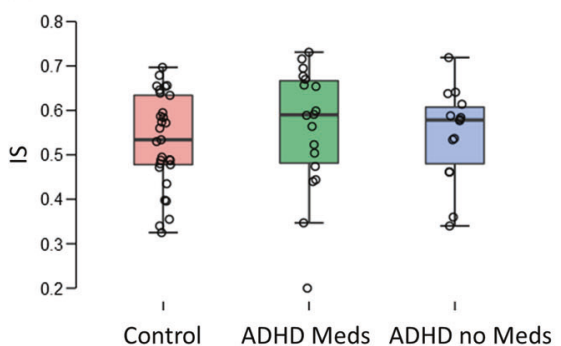

B

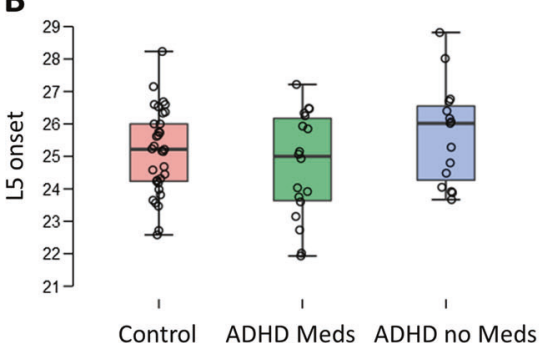

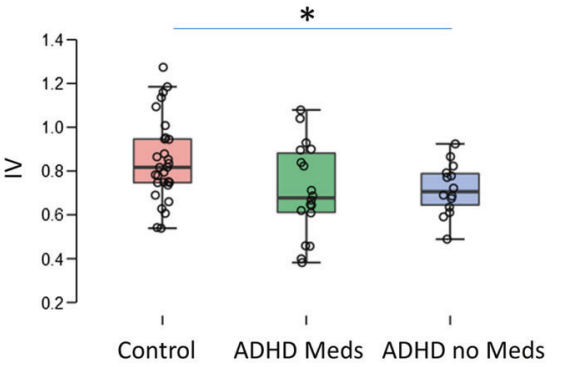
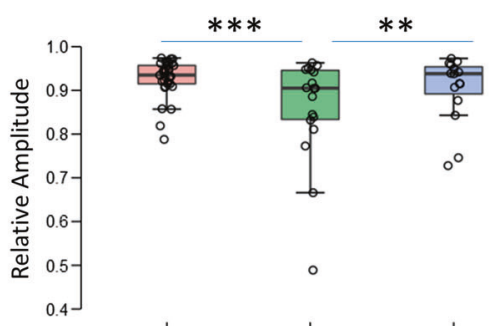

Control ADHD Meds ADHD no Meds
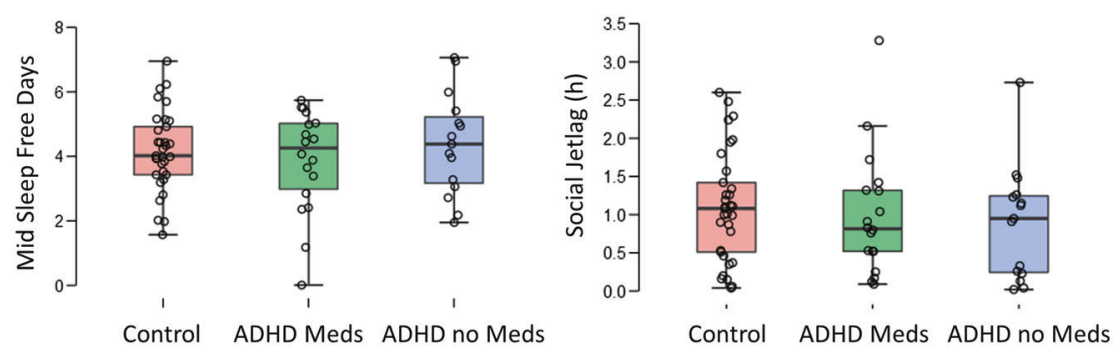

Fig. 1 Actigraphic measures of circadian rhythm robustness (a) and timing (b) in healthy controls, ADHD + medication and ADHD with no medication groups, displayed as boxplots and jittered data points. a There was no significant difference between groups for interdaily stability (IS), but intradaily variability (IV) was significantly higher in controls than in the ADHD no medication group. Relative amplitude was significantly lower in the ADHD + medication group than the control group. b There were no significant differences in the timing of the activity rhythm, as assessed by the time-of-onset of the least active $5 \mathrm{~h}$ period (L5 onset), the time of mid-sleep on free days or social jetlag. ${ }^{*} P<0.05,{ }^{* * *} P<0.001$ 

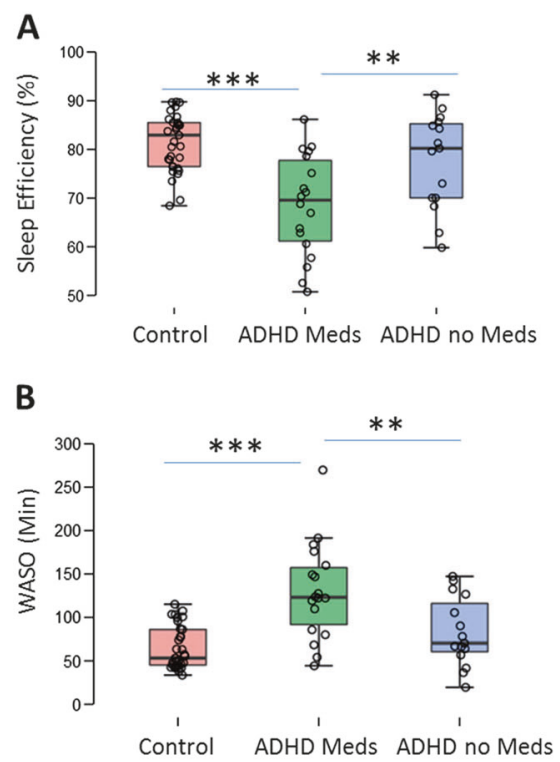
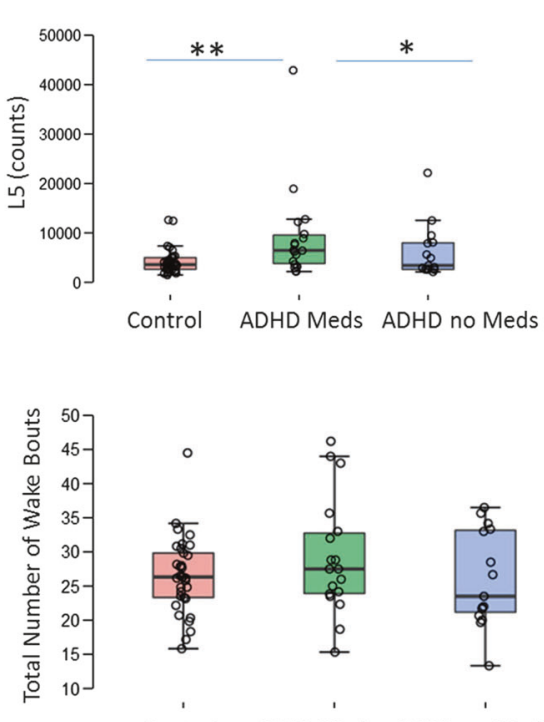

Control

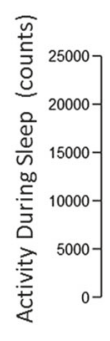

1201

Fig. 2 Actigraphic measures of sleep parameters, displayed as boxplots and jittered data points, across the three groups. a For measures of activity during sleep, there was significantly lower sleep efficiency in the ADHD + medication group compared to either the control or the ADHD no medication groups, and the ADHD + medication group had a greater amount of activity during L5 than the control group. b Looking at wakening during the night in more detail, the ADHD + medication group had more time spent in wakening after sleep onset (WASO), did not wake up more often, but when they did wake up the duration of the nocturnal wake bouts was longer. ${ }^{* *} P<0.01,{ }^{* * *} P<0.001$

effect of group on these variables (Pillai's trace $=0.263, F_{6,118}=$ $3.16, P=0.007$, partial ETA squared $=0.147$ ). When this effect was probed further it was revealed that IS did not vary between groups $(P=0.77)$, but IV $(P=0.026$, partial ETA squared $=0.122)$ and RA $(P=0.006$, partial ETA squared $=0.166)$ did. Bonferroni post-hoc tests showed that IV was lower in the ADHD + no medication group compared to healthy controls $(P=0.048)$, whilst RA was significantly lower in the ADHD + medication group compared to controls $(P=0.016)$. When ADHD symptom scores were controlled for in comparisons between the two ADHD groups, RA did vary according to medication status, with RA weaker in the ADHD + medication group than the ADHD no medication group $(P=0.008)$. These results indicate that the $\mathrm{ADHD}+$ medication group had more rhythm fragmentation, which was most likely due to the medication, whilst the ADHD no medication group showed better rhythm consolidation (via IV) than the control group. Next, actigraphic measures relating to sleep timing were analyzed (time of the onset of L5, the least active $5 \mathrm{~h}$ period, time of mid-sleep on weekend days, and social jetlag, the difference between mid-sleep on weekdays and weekend days; Fig. 1b). MANCOVA revealed no group-wise difference in these dependent variables (Pillai's trace $=0.058$, $F_{6,118}=0.59, P=0.72$ ).

Next, we examined activity during the sleep period. We grouped sleep efficiency, L5 (the activity during the least active $5 \mathrm{~h}$ ) and activity counts during sleep (Fig. 2a). MANCOVA showed a significant effect of group (Pillai's trace $=0.486, F_{6,118}=6.3, P<$ 0.001 , partial ETA squared $=0.24)$, with effects on sleep efficiency $(P<0.001$ partial ETA squared $=0.37)$ and L5 $(P=0.008$, partial ETA squared $=0.15)$, and activity during sleep $(P=0.014$, partial ETA squared $=0.13$ ). Sleep efficiency was lower in the ADHD + medication group than controls $(P<0.001)$ or the ADHD no medication group $(P=0.002$ controlling for ADHD symptom severity). L5 was higher in the ADHD + medication group than the control group $(P=0.003)$ and the ADHD no medication group $(P=0.029$, controlling for ADHD symptom severity). Total activity during sleep did not vary significantly between the two ADHD groups $(P=0.38)$, but was significantly elevated in the ADHD + meds group compared to control $(P=0.001)$. These results indicate that the ADHD + medication group had a higher level of activity during the night, and lower sleep efficiency than both controls and ADHD patients without medication. Subsequently, we examined parameters indicating the number of wakenings during the sleep period: WASO (time spent in wakening after sleep onset), number of wakenings, and the mean duration of wakenings (Fig. 2b). MANCOVA showed a significant effect of group on these dependent variables (Pillai's trace $=0.436, F_{6,116}=$ $5.47, P<0.001$, partial ETA squared $=0.218$ ). There were groupwise effects on WASO $(P<0.001$, partial ETA squared $=0.39)$ and average duration of wake bouts $(P<0.001$, partial ETA squared $=$ $0.39)$, but not on number of sleep bouts $(P=0.18)$. Post-hoc tests showed that the ADHD + medication group had higher WASO than controls $(P<0.001)$ and the $A D H D+$ no medication group ( $P=0.003$, controlling for ADHD symptoms), and longer duration wake bouts than controls $(P<0.001)$ or ADHD no medication $(P=$ $0.005)$. These results appear to indicate that medicated ADHD patients do not wake up more often during the night compared to controls and ADHD patients without medication, but that when they do they spend longer awake.

Ex-vivo clock gene expression in primary fibroblasts

The expression profiles of six clock genes were examined in primary fibroblasts cultured from skin biopsies and synchronized with dexamethasone (Fig. 3a). PER1 expression did not show a significant $\mathrm{ZT} \times$ group interaction via ANCOVA (controlling for sex and age; Greenhouse-Geisser corrected $F_{7.9,184}=1.98, P=0.054$ ). $P E R 2$ expression did show a significant $Z T \times$ group interaction (Greenhouse-Geisser corrected $F_{4.4,102}=3.86, P=0.019$ ). Post-hoc testing showed PER2 expression at ZTO to be significantly higher in the ADHD no medication group than controls $(P=0.004)$ and the ADHD medication group $(P=0.030)$. PER3 expression was strongly rhythmic in all three groups, but there was no $\mathrm{ZT} \times$ group interaction (Greenhouse-Geisser corrected $F_{3.4,82}, P=0.57$ ). There was a significant $\mathrm{ZT} \times$ group interaction term for $C R Y 1$ expression (Greenhouse-Geisser corrected $F_{4.9,113}=4.47, P=0.001$, partial ETA squared $=0.16$, with expression at ZT8 and ZT12 differing between groups; the ADHD no medication group differed to the control group $(P=0.010$ and $P=0.013$, respectively) and the ADHD + medication group (correcting for ADHD symptom severity, $P=0.021$ and $P=0.009$, respectively). There was no 

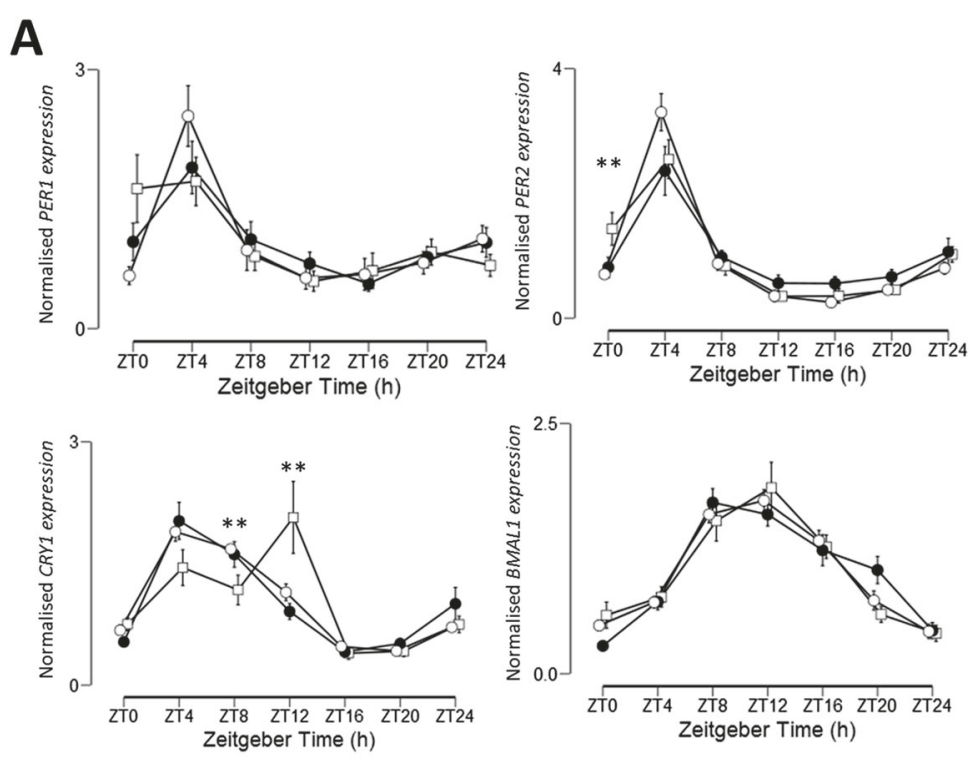
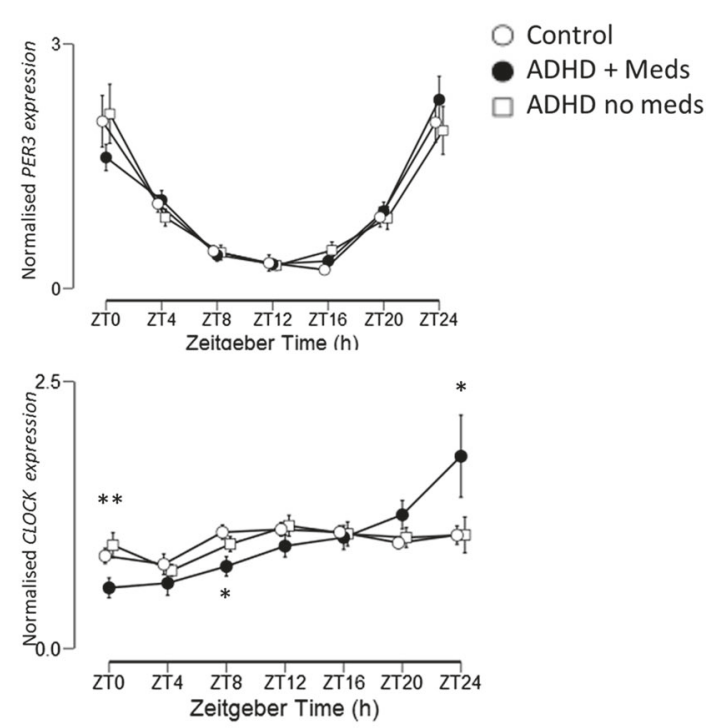

B
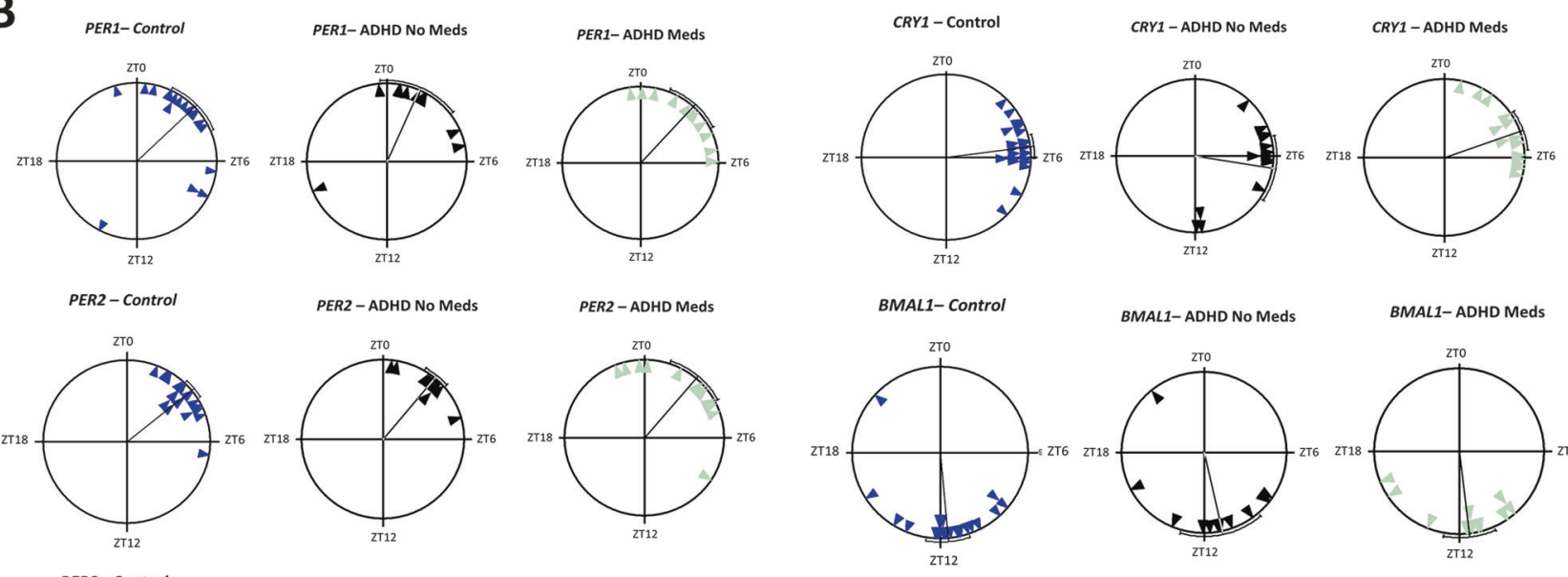

BMAL1- ADHD No Meds

BMAL1-ADHD Meds
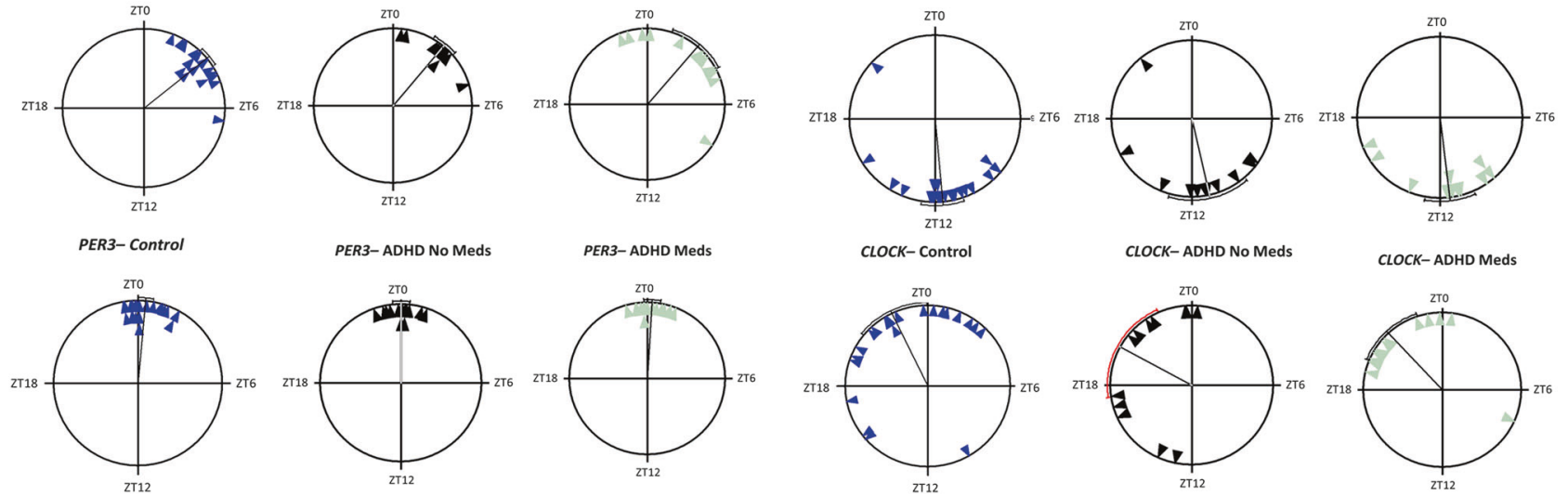

Fig. 3 Expression of clock genes in synchronized primary fibroblasts. a Expression profiles of PER1, PER2, PER3, CRY1, BMAL1, and CLOCK in primary fibroblasts cultured from controls (unfilled circles), patients with ADHD + medication (black circles) and patients with ADHD no medication (unfilled squares). ZT0 indicates time of culture synchronization. ${ }^{*} P<0.05,{ }^{* * *} P<0.001$ for post-hoc pairwise test. b Rayleigh plots of center-of-gravity for clock gene expression profiles derived from co-sinor fits. The diagonal line indicates mean center-of-gravity, and the curved line indicates the $95 \%$ confidence interval

ZT $\times$ group interaction for BMAL1 expression (Greenhouse-Geisser corrected $\left.F_{8.7,189}=1.26, P=0.27\right)$. There was a significant $\mathrm{ZT} \times$ group interaction for CLOCK expression (Greenhouse-Geisser corrected $F_{5.4,128}=3.2, P=0.008$ ), with expression significantly lower in the ADHD + medication group at ZTO than in the control and ADHD no medication groups $(P=0.045$ and $P=0.010$, respectively), lower in the $A D H D+$ meds group than controls $(P=0.006)$ and significantly higher at ZT24 in the ADHD + medication group compared to controls $(P=0.035)$. When the phase of expression of the clock genes was examined via cosinor analysis to identify the center-of-gravity of the fitted wave, there were no significant differences in the phase of PER1, PER2, PER3, $B M A L 1$, or CLOCK (Fig. 3b). There were significant differences in the phase of CRY1 expression between the ADHD + medication and the ADHD no medication groups (Watson-Williams $F$ test, $F_{1,27}=$ 4.49, $P=0.038)$.

Next, we examined the expression of a BMAL1:luc reporter in fibroblast cultures derived from control, ADHD + medication and ADHD no medication groups (Fig. 4). There were no significant differences in either the period or the amplitude of the $B M A L 1:$ Iuc rhythm in these cultures following synchronization (Fig. $4 \mathrm{~b} ; F_{2,13}=0.49, P=0.64$ for period and $F_{2,13}=2.42, P=0.10$ for amplitude via ANCOVA with age and sex as covariates). When the phase of the rhythm was examined, it was found that the peak of expression on the 1st day after synchronization occurred later in the ADHD no medication group compared to both the healthy 
A

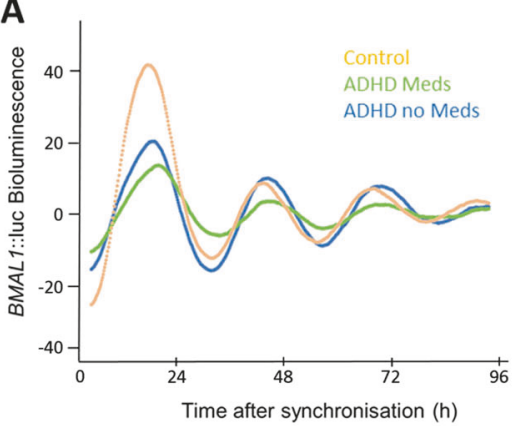

\section{B}

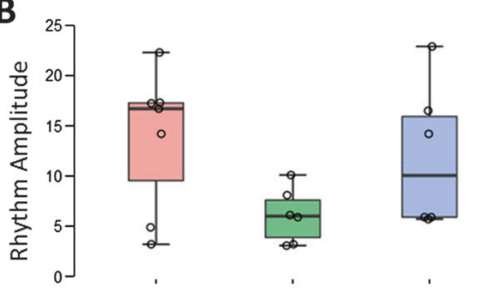

C
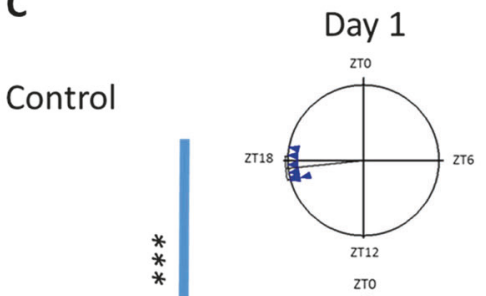

ADHD

Meds

Control ADHD Meds ADHD no Meds

ADHD

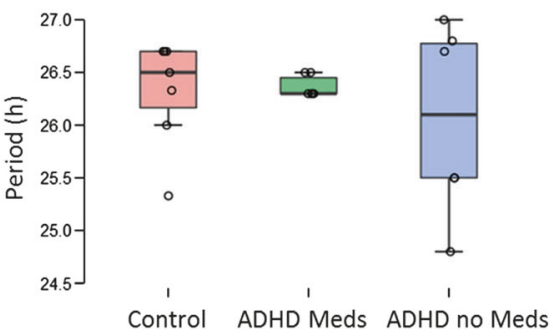

no Meds
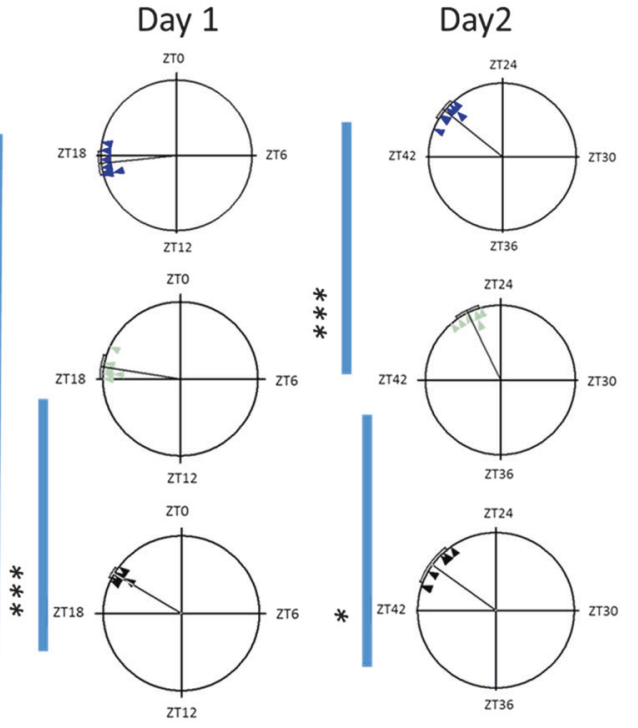

Day3
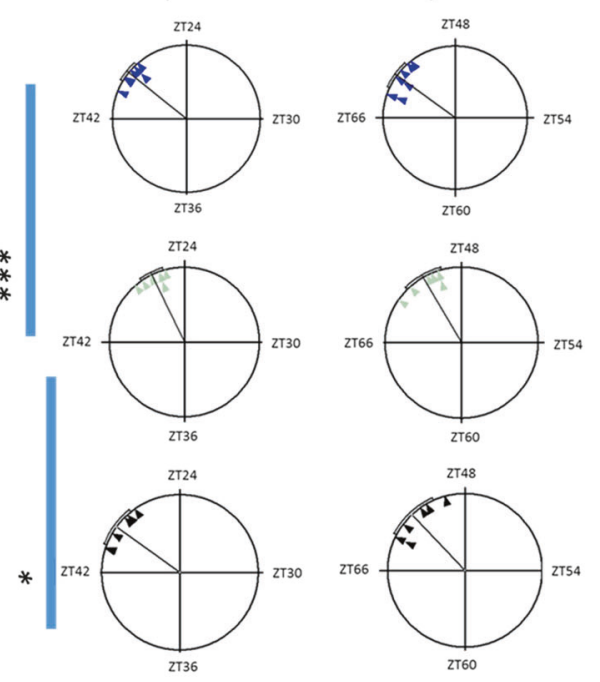

Fig. 4 Expression of BMAL1:IUc in fibroblasts from controls and ADHD patients. a Bioluminescence from the BMAL1:Iuc reporter in controls (orange), ADHD + medication (green), and ADHD with no medication (blue). $\mathbf{b}$ Boxplots representing the rhythm amplitude and period of the bioluminescence from BMAL1:luc transformed fibroblasts. There were no significant differences. c Rayleigh plots for the acrophases of bioluminescence on each of the first 3 days following synchronization. ${ }^{* *} P<0.001,{ }^{*} P<0.05$. $N=7$ for controls, $N=6$ for both ADHD + medication and ADHD no medication

controls and the ADHD + medication groups $(P<0.001$ for both via Watson-Williams F-test; Fig. 4c). On the 2nd day after synchronization, the ADHD + medication group's acrophase was significantly later than both the control and ADHD no medication groups $(P<0.001$ and $P<0.01$, respectively). On the 3rd day after synchronization, there were no significant differences in the acrophases between groups. These results indicate that the phase of BMAL 1:luc expression following a dexamethasone pulse appears to be influenced by ADHD and medication status.

\section{DISCUSSION}

The results of the present study illustrate that both ADHD and medication status impact on circadian function. We demonstrate that ADHD medication status has a significant impact on a number of behavioral circadian rhythms and sleep outcomes. Clock gene expression data indicate an impact of both ADHD and medication status on the core molecular clockworks. The ex-vivo nature of the molecular assays used indicates that medication status may elicit persistent changes in circadian function in ADHD patients.

A number of previous studies have indicated that circadian processes are altered in ADHD patients. For example, Baird et al. [17] reported that adults with ADHD showed a blunting of rhythms of PER2 and BMAL1 expression in oral mucosa, delayed cortisol rhythms relative to wake-time, increased nocturnal activity, and increased eveningness. However, this study did not differentiate between medicated and un-medicated ADHD patients. Van Veen et al. [19] reported phase-delay of the circadian system in adult ADHD patients not using medication. The impact of stimulants on sleep has previously been reported; for example, a RCT in adults with ADHD showed that methylphenidate treatment results in later bedtime and sleep onset, and shorter but more consolidated sleep [32]. Further, preclinical studies demonstrate that ADHD medication can impact on circadian processes: Antle et al. [21] reported that chronic treatment of mice with methylphenidate leads to a delay of sleep onset, a lengthening of free-running circadian period and alters the neuronal excitability of SCN neurons. These findings which are consistent with the idea that methylphenidate may promote sleep-onset insomnia. Methylphenidate has also been shown to alter diurnal activity rhythms in rats [33]. A recent report in the diurnal rodent Arvicanthis ansorgei also indicates that chronic methylphenidate treatment leads to behavioral phase delays, indicating that methylphenidate can impact on rhythms in both nocturnal and diurnal rodents [24]. The non-stimulant atomoxetine has been shown to induce phase-delays of free-running rhythms in mice, as well as modulating photic-resetting of the circadian clock [22]. Both methylphenidate and atomoxetine have been shown to alter diurnal rhythms of clock gene expression across a number of brain areas in mice [23]. Therefore, there is an a-priori basis to believe that ADHD medication (either stimulant or non-stimulant) may impact on circadian rhythm processes in ADHD patients. Current findings from the actigraphic data indicate that many of the observed differences may be due to medication status, rather than ADHD per se. We observe a decrease of activity rhythm fragmentation in ADHD patients using medication (as measured by IV), a finding that has previously been reported [32]. 
We also report a decrease in the rhythm amplitude, as assessed by RA in patients with ADHD using medication compared to both controls and un-medicated ADHD patients; therefore it may be that medication is lowering rhythm amplitude by inducing increased levels of nocturnal activity. This is supported by the finding that $L 5$ activity (typically from the $5 \mathrm{~h}$ period between near midnight and near 5 a.m.) is increased in medicated ADHD patients, compared to controls and un-medicated ADHD patients. This increase in nocturnal activity likely results in the decrease in sleep efficiency observed in medicated ADHD participants. Medication is also associated with increased duration of average nocturnal wake bouts, although not with more frequent wakening, with the overall effect of greater time spent in wakening after sleep onset. This finding is at variance from the findings of Boonstra et al. [32], who reported decreases in both frequency and duration of nocturnal wake bouts with methylphenidate treatment. Differences in study design (e.g., RCT vs. observational study) may account for such a discrepancy. It is noticeable that neither ADHD or medication status differences were observed in measures of rest-activity rhythm timing and social jetlag; previous results also did not show changes in activity rhythm timing (e.g., measured by time of onset of L5 [17]), although phase markers such as DLMO indicate a phase-delayed clock [19] and ADHD patients show a marked shift towards in diurnal preference towards eveningness (e.g., Rybak et al. [18]). As diurnal preference may reflect both underlying phase of entrainment along with psychological factors [16, 34], the observed shift to eveningness in ADHD might primarily reflect an increased psychological preference rather than changes at the neurobiological or molecular levels (although of course such systems may be recruited indirectly to amplify such psychological changes).

Ex-vivo expression of clock genes in fibroblast has previously been demonstrated to be a useful approach to the study of circadian processes associated with sleep/wake behavior (e.g., $[29,35])$ and psychiatric disorders (e.g., bipolar disorder [36] and schizophrenia [37]). Ex-vivo fibroblasts have also been used in other aspects of ADHD research [38]. An interesting facet of using ex-vivo fibroblast cultures is that effects of current medication on clock processes may not be acute, and may be more reflective of long-lasting plastic changes possibly through epigenetic modifications $[39,40]$. We report a number of changes in the rhythmic expression of clock genes. Cultures from ADHD patients with no medication showed higher expression of PER2 immediately after synchronization than either controls or medicated ADHD cultures, perhaps reflecting greater acute response to the dexamethasone pulse in these cells. Expression of CLOCK was altered in the $\mathrm{ADHD}+$ medication cultures at ZTO and ZT24. The most striking changes were in the expression of $C R Y 1$; cultures from unmedicated ADHD patients showed delayed peak in expression. Data from cell cultures transfected with the BMAL1:luc reporter indicates that the rate of synchronization of BMAL1:luc rhythms following a dexamethasone pulse varies across the three groups, with cultures from ADHD patients with no medication seeming to display an acrophase on day 1 in culture following the synchronizing pulse that is similar to that on day 3 , whilst both controls and ADHD + medication cultures show an earlier peak on day 1 which delays on subsequent days in culture. Data from the $B M A L: I U c$ assay has greater temporal resolution and allows for changes to be observed that might not be apparent from more expression profiles from qRT-PCR samples taken every $4 \mathrm{~h}$.

It is not clear why some components of the clock gene cycle show differences in expression, and some do not; however, this has previously been shown in fibroblasts from patients with schizophrenia, where changes in mRNA cycling occur for PER2, $C R Y 1$, and CLOCK [37]. These are the same genes that exhibit differences in the current study. A previous study in adults with ADHD demonstrated changes in PER2 and BMAl1 expression in the oral mucosa [17]. It is interesting to note that a polymorphism in
CLOCK (3111T/C) that affects the expression of PER2 [41] has been associated with ADHD and ADHD symptoms in a number of studies [42-44]. Treatment status may also impact on clock gene rhythms, as sub-chronic treatment with methylphenidate and atomoxetine alters clock gene expression patterns in mice [23], and psychostimulants in general are well described as altering clock gene expression and circadian processes [45]. One interpretation of the current data is that aspects of the molecular clock systems are affected by ADHD status (at the gene expression level at least; e.g., PER2 and CRY1 expression), and medication may normalize these expression patterns, whilst also resulting in some deviation in CLOCK expression. Some of these changes may be as a result of altered synchronization within cultures following dexamethasone pulses, as indicated by the BMAL1:luc data. Future work may address the impact of such changes on rhythms of global gene expression in ADHD via transcriptomic analysis, and may also address whether changes in mRNA translate into changes in clock gene protein product expression.

Overall, our data support the dual contention that both ADHD status and ADHD medication impact on circadian processes and sleep, highlighting the need to address both the effect of the underlying condition and the ongoing management of the condition on the circadian clock. There are a number of caveats to consider in the interpretation of our results: the first is the relatively modest sample size, and inherent heterogeneity within the ADHD groups. Further, the subsample for the BMAL1::/uc analysis was smaller than for the Q-PCR analysis, although the much greater temporal resolution of the bioluminescence monitoring should result in an ability to detect more subtle effects compared to static time-series sampling. Pharmacotherapy regimes in the treated $A D H D$ groups were not universal within the group, with a small number of patients treated with atomoxetine; however, we do believe that our sample was clinically representative. Notwithstanding these considerations, our data may have clinical implications for how sleep disturbances in ADHD in adults are considered and addressed: firstly, we highlight the key importance of taking ADHD medication into account when assessing sleep disturbances. Interestingly, chronotype has been shown to moderate tolerability and side-effect profile on sleep and mood of other psychoactive medications, such as levetiracetam in epilepsy, with morning types showing the lowest levels of toleration [46]. The impact of methylphenidate or atomoxetine on sleep may be moderated through chronotype, and future studies should address this possibility. Second, the formulation (extended vs. immediate release) and time of dosing may also moderate the impact of ADHD medications on sleep and circadian rhythms. A careful assessment of sleep patterns and chronotype of patients before initiation of pharmacotherapy might be used to inform time of optimal dosing for individuals. Finally, where sleep disturbances persist in ADHD patients for whom it is otherwise desirable to continue with pharmacotherapy, or indeed in those patients not using ADHD medication but experiencing sleep disturbances, consideration could be given to psychotherapeutic and behavioral interventions to address sleep and circadian disturbances. For example, pairing of cognitive behavioral therapy for insomnia and/or light chronotherapy may be useful in improving sleep outcomes, and possibly ADHD symptoms as in general addressing sleep problems lead to primary symptom improvement across a number of psychiatric conditions $[47,48]$.

\section{FUNDING AND DISCLOSURE}

Johannes Thome has received financial support from pharmaceutical companies (Actelion, Astra Zeneca, Bristol-Myers Squibb, EVER Neuro Pharma GmbH, Janssen-Cilag, Lilly, Lundbeck, MEDICE, Merz, Novartis, Pfizer, Roche, Servier, Shire, Trommsdorff) some of which manufacture medication used in the treatment of ADHD patients. Michael Colla received lecture fees from Eli Lilly, 
Janssen-Cilag, and Shire, acted as a consultant to Eli Lilly, JanssenCilag, and Shire, and received grants from the BMBF (Federal Ministry of Education and Research), the BMWi (Federal Ministry of Economics and Technology), and the EU (European Union). Andrew Coogan, Maria Schenk, Denise Palm, Adriana Uzoni, Jana Grube, Anthony Tsang, Isa Kolbe, Niall McGowan, Roland Wandschneider, Henrik Oster, and Frank Faltraco have no potential conflicts of interest to disclose.

\section{ADDITIONAL INFORMATION}

Supplementary Information accompanies this paper at (https://doi.org/10.1038/ s41386-019-0327-6).

Publisher's note: Springer Nature remains neutral with regard to jurisdictional claims in published maps and institutional affiliations.

\section{REFERENCES}

1. Willcutt EG. The prevalence of DSM-IV attention-deficit/hyperactivity disorder: a meta-analytic review. Neurotherapeutics. 2012;9:490-9. https://doi.org/10.1007/ s13311-012-0135-8

2. Sibley MH, Mitchell JT, Becker SP. Method of adult diagnosis influences estimated persistence of childhood ADHD: a systematic review of longitudinal studies. Lancet Psychiatry. 2016;3:1157-65. https://doi.org/10.1016/S2215-0366 (16)30190-0

3. Brevik EJ, Lundervold AJ, Halmøy A, Posserud M-B, Instanes JT, Bjorvatn B, et al. Prevalence and clinical correlates of insomnia in adults with attention-deficit hyperactivity disorder. Acta Psychiatr Scand. 2017;136:220-7. https://doi.org/ 10.1111/acps.12756

4. Hennig T, Krkovic K, Lincoln TM. What predicts inattention in adolescents? An experience-sampling study comparing chronotype, subjective, and objective sleep parameters. Sleep Med. 2017;38:58-63. https://doi.org/10.1016/j. sleep.2017.07.009

5. Logan RW, Hasler BP, Forbes EE, Franzen PL, Torregrossa MM, Huang $\mathrm{YH}$, et al., et al. Impact of sleep and circadian rhythms on addiction vulnerability in adolescents. Biol Psychiatry. 2018;83:987-96. https://doi.org/10.1016/j.biopsych.2017.11.035

6. McGowan NM, Coogan AN. Sleep and circadian rhythm function and trait impulsivity: an actigraphy study. Psychiatry Res. 2018;268:251-6. https://doi.org/ 10.1016/j.psychres.2018.07.030

7. Wynchank D, Bijlenga D, Beekman AT, Kooij JJS, Penninx BW. Adult attentiondeficit/hyperactivity disorder (ADHD) and insomnia: an update of the literature. Curr Psychiatry Rep. 2017;19:98 https://doi.org/10.1007/s11920-017-0860-0

8. Krause AJ, Simon EB, Mander BA, Greer SM, Saletin JM, Goldstein-Piekarski AN, et al. The sleep-deprived human brain. Nat Rev Neurosci. 2017;18:404 https://doi. org/10.1038/nrn.2017.55

9. Scammell $T E$, Arrigoni $E$, Lipton J. Neural circuitry of wakefulness and sleep. Neuron. 2017;93:747-65. https://doi.org/10.1016/j.neuron.2017.01.014

10. Borbély AA, Daan S, Wirz-Justice A, Deboer T. The two-process model of sleep regulation: a reappraisal. J Sleep Res. 2016;25:131-43. https://doi.org/10.1111/ jsr.12371

11. Pilorz V, Helfrich-Förster $C$, Oster $H$. The role of the circadian clock system in physiology. Pflügers Arch. 2018;470:227-39. https://doi.org/10.1007/s00424-0172103-y

12. Takahashi JS. Transcriptional architecture of the mammalian circadian clock. Nat Rev Genet. 2017;18:164-79. https://doi.org/10.1038/nrg.2016.150

13. Zhang $\mathrm{R}$, Lahens NF, Ballance $\mathrm{HI}$, Hughes ME, Hogenesch JB. A circadian gene expression atlas in mammals: implications for biology and medicine. Proc Natl Acad Sci USA. 2014;111:16219-24. https://doi.org/10.1073/pnas.1408886111.

14. Hughes S, Jagannath A, Hankins MW, Foster RG, Peirson SN. Chapter Six-photic regulation of clock systems. In: Sehgal A, editor. Methods in enzymology, vol. 552. Academic Press, London; 2015. p. 125-43. https://doi.org/10.1016/bs. mie.2014.10.018.

15. Zaki NFW, Spence DW, BaHammam AS, Pandi-Perumal SR, Cardinali DP, Brown GM. Chronobiological theories of mood disorder. Eur Arch Psychiatry Clin Neurosci. 2018;268:107-18. https://doi.org/10.1007/s00406-017-0835-5

16. Coogan AN, McGowan NM. A systematic review of circadian function, chronotype and chronotherapy in attention deficit hyperactivity disorder. Atten Defic Hyperact Disord. 2017;9:129-47. https://doi.org/10.1007/s12402-016-0214-5

17. Baird AL, Coogan AN, Siddiqui A, Donev RM, Thome J. Adult attention-deficit hyperactivity disorder is associated with alterations in circadian rhythms at the behavioural, endocrine and molecular levels. Mol Psychiatry. 2012;17:988-95. https://doi.org/10.1038/mp.2011.149
18. Rybak YE, McNeely HE, Mackenzie BE, Jain UR, Levitan RD. Seasonality and circadian preference in adult attention-deficit/hyperactivity disorder: clinical and neuropsychological correlates. Compr Psychiatry. 2007;48:562-71. https://doi. org/10.1016/j.comppsych.2007.05.008

19. Van Veen MM, Kooij JJS, Boonstra AM, Gordijn MCM, Van Someren EJW. Delayed circadian rhythm in adults with attention-deficit/hyperactivity disorder and chronic sleep-onset insomnia. Biol Psychiatry. 2010;67:1091-6. https://doi.org/ 10.1016/j.biopsych.2009.12.032

20. Santhi N, Lazar AS, McCabe PJ, Lo JC, Groeger JA, Dijk D-J. Sex differences in the circadian regulation of sleep and waking cognition in humans Proc Natl Acad Sci USA. 2016;113:E2730-9. https://doi.org/10.1073/pnas.1521637113.

21. Antle MC, van Diepen HC, Deboer T, Pedram P, Pereira RR, Meijer JH. Methylphenidate modifies the motion of the circadian clock. Neuropsychopharmacology. 2012;37:2446 https://doi.org/10.1038/npp.2012.103

22. O'Keeffe SM, Thome J, Coogan AN. The noradrenaline reuptake inhibitor atomoxetine phase-shifts the circadian clock in mice. Neuroscience. 2012;201:219-30. https://doi.org/10.1016/j.neuroscience.2011.11.002

23. Baird AL, Coogan AN, Kaufling J, Barrot M, Thome J. Daily methylphenidate and atomoxetine treatment impacts on clock gene protein expression in the mouse brain. Brain Res. 2013;1513:61-71. https://doi.org/10.1016/j.brainres.2013.03.038

24. Mendoza J, van Diepen HC, Pereira RR, Meijer JH. Time-shifting effects of methylphenidate on daily rhythms in the diurnal rodent Arvicanthis ansorgei. Psychopharmacology. 2018;235:2323-33. https://doi.org/10.1007/s00213-0184928-2

25. Morash-Conway J, Gendron M, Corkum P. The role of sleep quality and quantity in moderating the effectiveness of medication in the treatment of children with ADHD. Atten Defic Hyperact Disord. 2017;9:31-8. https://doi.org/10.1007/s12402016-0204-7

26. Takashima, A. Establishment of Fibroblast Cultures. Current Protocols, 1999;1:2.1.1-2.1.12

27. Kushida CA, Chang A, Gadkary C, Guilleminault C, Carrillo O, Dement WC. Comparison of actigraphic, polysomnographic, and subjective assessment of sleep parameters in sleep-disordered patients. Sleep Med. 2001;2:389-96. https://doi. org/10.1016/S1389-9457(00)00098-8

28. Tsang AH, Sanchez-Moreno C, Bode B, Rossner MJ, Garaulet M, Oster H. Tissuespecific interaction of Per1/2 and Dec2 in the regulation of fibroblast circadian rhythms. J Biol Rhythms. 2012;27:478-89. https://doi.org/10.1177/ 0748730412462838

29. Hida A, Ohsawa Y, Kitamura S, Nakazaki K, Ayabe N, Motomura Y, et al. Evaluation of circadian phenotypes utilizing fibroblasts from patients with circadian rhythm sleep disorders. Transl Psychiatry. 2017;7:e1106 https://doi.org/10.1038/ tp.2017.75

30. O'Keeffe SM, Beynon AL, Davies J, Moynagh PN, Coogan AN. NF-kB signalling is involved in immune-modulation, but not basal functioning of the mouse suprachiasmatic circadian clock. Eur J Neurosci. 2017;45:1111-23.

31. Cohen J. Statistical power analysis for the behavioral sciences. Taylor and Francis, New York; 1988.

32. Boonstra AM, Kooij JJ, Oosterlaan J, Sergeant JA, Buitelaar JK, Van Someren EJ. Hyperactive night and day? Actigraphy studies in adult ADHD: a baseline comparison and the effect of methylphenidate. Sleep. 2007;30:433-42.

33. Trinh T, Kohllepin S, Yang PB, Burau KD, Dafny N. Adult female rats altered diurnal locomotor activity pattern following chronic methylphenidate treatment. J Neural Transm. 2013;120:1717-31. https://doi.org/10.1007/s00702-013-1063-4

34. McGowan NM, Brannigan R, Doyle D, Coogan AN. Diurnal preference, circadian phase of entrainment and time perspectives: just what are the relationships? Pers Indiv Differ. 2017;112:79-84. https://doi.org/10.1016/j.paid.2017.02.051

35. Brown SA, Fleury-Olela F, Nagoshi E, Hauser $C$, Juge $C$, Meier CA, et al. The period length of fibroblast circadian gene expression varies widely among human individuals. PLoS Biol. 2005;3:e338 https://doi.org/10.1371/journal.pbio.0030338

36. McCarthy MJ, Wei H, Marnoy Z, Darvish RM, McPhie DL, Cohen BM, et al. Genetic and clinical factors predict lithium's effects on PER2 gene expression rhythms in cells from bipolar disorder patients. Transl Psychiatry. 2013;3:e318 https://doi.org/ 10.1038/tp.2013.90

37. Johansson A-S, Owe-Larsson B, Hetta J, Lundkvist GB. Altered circadian clock gene expression in patients with schizophrenia. Schizophr Res. 2016;174:17-23. https://doi.org/10.1016/j.schres.2016.04.029

38. Johansson J, Landgren M, Fernell E, Vumma R, Åhlin A, Bjerkenstedt L, et al. Altered tryptophan and alanine transport in fibroblasts from boys with attentiondeficit/hyperactivity disorder (ADHD): an in vitro study. Behav Brain Funct. 2011;7:40. https://doi.org/10.1186/1744-9081-7-40

39. Csoka AB, Szyf M. Epigenetic side-effects of common pharmaceuticals: a potential new field in medicine and pharmacology. Med Hypotheses. 2009;73:770-80. https://doi.org/10.1016/j.mehy.2008.10.039

40. Ding K, Yang J, Reynolds GP, Chen B, Shao J, Liu R, et al. DAT1 methylation is associated with methylphenidate response on oppositional and hyperactive- 
impulsive symptoms in children and adolescents with ADHD. World J Biol Psychiatry. 2017;18:291-9. https://doi.org/10.1080/15622975.2016.1224928

41. Ozburn AR, Purohit K, Parekh PK, Kaplan GN, Falcon E, Mukherjee S, et al. Functional implications of the CLOCK 3111T/C single-nucleotide polymorphism. Front Psychiatry. 2016;7:67 https://doi.org/10.3389/fpsyt.2016.00067

42. Kissling C, Retz W, Wiemann S, Coogan AN, Clement RM, Hünnerkopf R, et al. A polymorphism at the $3^{\prime}$-untranslated region of the CLOCK gene is associated with adult attention-deficit hyperactivity disorder. Am J Med Genet B Neuropsychiatr Genet. 2008;147:333-8. https://doi.org/10.1002/ajmg.b.30602

43. Xu X, Breen G, Chen C-K, Huang $Y-S, W u ~ Y-Y$, Asherson P. Association study between a polymorphism at the 3'-untranslated region of CLOCK gene and attention deficit hyperactivity disorder. Behav Brain Funct. 2010;6:48. https://doi. org/10.1186/1744-9081-6-48

44. Jeong SH, Yu J-C, Lee CH, Choi K-S, Choi J-E, Kim SH, et al. Human CLOCK geneassociated attention deficit hyperactivity disorder-related features in healthy adults: quantitative association study using Wender Utah Rating Scale. Eur Arch Psychiatry Clin Neurosci. 2014;264:71-81. https://doi.org/10.1007/s00406-0130443-y

45. Mendoza J, Challet E. Circadian insights into dopamine mechanisms. Neuroscience. 2014;282:230-42. https://doi.org/10.1016/j.neuroscience.2014.07.081

46. Taneja R, Hunter K, Burakgazi-Dalkilic E, Carran M. Effect of sleep patterns on levetiracetam induced mood changes. Epilepsy Behav. 2017;75:237-40. https:// doi.org/10.1016/j.yebeh.2017.07.038

47. Freeman D, Sheaves B, Goodwin GM, Yu LM, Nickless A, Harrison PJ. The effects of improving sleep on mental health (OASIS): a randomised controlled trial with mediation analysis. Lancet Psychiatry. 2017;4:749-58. https://doi.org/10.1016/ S2215-0366(17)30328-0

48. Jansson-Fröjmark $M$, Norell-Clarke A. Cognitive behavioural therapy for insomnia in psychiatric disorders current. Sleep Med Rep. 2016;2:233-40. https://doi.org/ 10.1007/s40675-016-0055-y 